\title{
Penetration of newer quinolones in the empyema fluid
}

\author{
I.E. Liapakis*,\#,+, I. Kottakis\#, M.N. Tzatzarakis", A.M. Tsatsakis", M.S. Pitiakoudis*, P. Ypsilantis*, \\ R.W. Light ${ }^{+}$, C.E. Simopoulos*, D.E. Bouros ${ }^{\#}$
}

Penetration of newer quinolones in the empyema fluid. I.E. Liapakis, I. Kottakis, M.N. Tzatzarakis, A.M. Tsatsakis, M.S. Pitiakoudis, P. Ypsilantis, R.W. Light, C.E. Simopoulos, D.E. Bouros. (C) ERS Journals Ltd 2004.

ABSTRACT: The degree of penetration of newer quinolones into the pleural fluid has not been studied. The objective of the present study was to determine the degree to which moxifloxacin and levofloxacin penetrate into empyemic pleural fluid using a new rabbit model of empyema.

An empyema was created via the intrapleural injection of turpentine $(1 \mathrm{~mL})$, followed $24 \mathrm{~h}$ later by instillation of $2 \mathrm{~mL}\left(1 \times 10^{10}\right)$ Escherichia coli bacteria (ATCC 35218) into the pleural space of New Zealand white rabbits. After an empyema was verified by thoracentesis and pleural fluid analysis, moxifloxacin and levofloxacin $\left(25 \mathrm{mg} \cdot \mathrm{kg}^{-1}\right.$ for both, $i$.v.) were administered. Antibiotic levels were determined in samples of pleural fluid and in blood collected serially over $12 \mathrm{~h}$. Antibiotic levels were measured using HPLC.

Each of the antibiotics penetrated well into the empyemic pleural fluid. Antibiotic penetration was the greatest for moxifloxacin (area under the curve (AUC) for pleural fluid/blood (AUCPF/AUCblood) ratio=1.37) followed by levofloxacin (ratio=1.13). The time to equilibration between the pleural fluid and blood antibiotic levels was more rapid for moxifloxacin $(3.9 \mathrm{~h})$ than for levofloxacin $(4.4 \mathrm{~h})$. With moxifloxacin, the peak pleural fluid concentration $(\mathrm{Cmax}, \mathrm{PF})$ was $2.77 \mu \mathrm{g} \cdot \mathrm{mL}^{-1}$ and occurred at a time to maximum pleural fluid concentration $\left(T_{\max }, \mathrm{PF}\right)$ of $6 \mathrm{~h}$ after infusion and decreased thereafter. The peak blood concentration (Cmax,blood) was $4.81 \mu \mathrm{g} \cdot \mathrm{mL}^{-1}$ at $1 \mathrm{~h}$ after administration. With levofloxacin, the peak pleural fluid level $\left(\mathrm{Cmax}, \mathrm{PF}=1.39 \mu \mathrm{g} \cdot \mathrm{mL}^{-1}\right)$

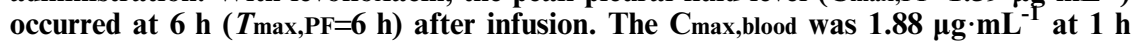
after administration.

In conclusion, differences were found in the degree of penetration of the two quinolones into infected pleural fluid in rabbits. The clinical significance of these differences is unknown. More studies are needed to evaluate the pharmacokinetic parameters in the pleural space in humans.

Eur Respir J 2004; 24: 466-470. *2nd Dept of Surgery and "Pneumonology, Medical School, Democritus University of Thrace, Alexandroupolis, and Center of Toxicological Science and Research, Dept of Medicine, University of Crete, Heraklion, Greece. ${ }^{+}$Pulmonary Dept, Saint Thomas Hospital and Vanderbilt University, Nashville, TN, USA.

Correspondence: D.E. Bouros, Medical School, University of Thrace, Head, Dept of Pneumonology, University Hospital, Alexandroupolis 68100, Greece.

Fax: 302551076106

E-mail: bouros@med.duth.gr

Keywords: Chromatography

Escherichia coli

levofloxacin

moxifloxacin

plural empyema

turpentine

Received: March 42004

Accepted after revision: May 242004

This study was supported by a grant from Abbott Laboratories (Hellas) and ELPEN SA Pharmaceuticals.
Bacterial pneumonia has an associated parapneumonic pleural effusion in $\sim 40 \%$ of cases [1]. A small percentage of these parapneumonic effusions evolve into complicated parapneumonic effusions and empyema [2, 3]. Thoracic empyema continues to remain a significant medical problem. There are several therapeutic options available and the choice of therapy is usually dictated by the severity of the disease on presentation. The usual initial treatment remains parenteral antibiotics with chest tube placement $[4,5]$. Although numerous antibiotics are used for the treatment of pneumonia with parapneumonic effusion, little has been published on the pharmacokinetics of parenterally administered antibiotics in the pleural fluid. Advanced-generation fluoroquinolone agents offer excellent activity against both Gram-positive and Gramnegative bacilli. Fluoroquinolones variably offer greater efficacy with highly resistant pneumococcii, and/or greater anaerobic coverage, providing advantages for treating selected patients when used as monotherapy. Some medical systems have adopted fluoroquinolones as empirical therapy in clinical pathways for the treatment of community-acquired pneumonia $[6,7]$. The quinolones are derivatives of nalidixic acid and exhibit favourable-pharmacokinetic properties. Thus, the new quinolones, such as levofloxacin and moxifloxacin show promise for treatment of a variety of bacterial infections [8,9].
The most frequent microbes in pleural empyema are anaerobic bacteria, Streptococcus pneumoniae, group A $\beta$-haemolytic streptococci and Staphylococcus aureus [10, 11]. Importantly, empirical therapy needs to be initiated as soon as pleural fluid, sputum and blood samples have been taken. Second and third generation cephalosporins, $\beta$-lactam- $\beta$-lactamase inhibitor combinations, macrolides, fluoroquinolones, metronidazole, clindamycin, imipenem, aminoglycosides or aztreonam may be considered [12].

In addition to the specificity of the antimicrobial agent for the offending microorganism, its distribution within the body is a critical factor in determining its therapeutic efficacy. If the antimicrobial agent does not enter the site at which the offending microorganism resides, bacterial growth will continue despite in vitro susceptibility of the organism to the drug. The utility of an antimicrobial agent in the treatment of pleural diseases depends on the attainment of adequate drug concentrations within intrapleural sites of infection [12]. In fact, specific to the treatment of pleural infections, it is important to obtain sufficient levels of the antibiotics in the pleural fluid. For various reasons, such as limited permeability of thickened pleura, pleural fluid antibiotic levels obtained after systemic administration could be different compared with serum levels. However, there have been only 
limited studies on the relationship between serum and pleural fluid antibiotic levels. Most studies in humans have involved patients with diseases other than empyema. The physiology is likely to be different with empyema, since empyema fluid is more acidic and the pleural surfaces are thicker [13].

Correlations between the pleural fluid and serum antibiotic levels have not been studied in detail in animals. TEIXEIRA et al. [14], in an elegant study using a new rabbit model of empyema, determined the relationships between the pleural fluid and serum antibiotic levels of metronidazole, penicillin, clindamycin, ceftriaxone, vancomycin and gentamicin. The pharmacokinetics were studied after $i$. v. administration. It was found that the penetration of these antibiotics into the infected pleural fluid, and the equilibration between the serum and pleural fluid varied from one antibiotic to the next.

The purpose of the present study was to determine the pharmacokinetic parameters of moxifloxacin and levofloxacin in the blood and pleural fluid in an experimental rabbit model of empyema, after i.v. administration. It was hypothesised that both moxifloxacin and levofloxacin would penetrate well into the pleural fluid and achieve therapeutic levels in the pleural fluid of rabbits with empyema.

\section{Materials and methods}

\section{Animals}

A total of 10 male New Zealand white rabbits $(4.0-5.0 \mathrm{~kg}$ ) were used for the study. The animals were housed in individual cages and allowed food and water ad libitum. Three rabbits each were used to study the pleural fluid antibiotic levels after $i$.v. administration of the antibiotics moxifloxacin and levofloxacin. This project was conducted in the Experimental Surgery Dept, Medical School, University of Thrace, Alexandroupolis, Greece, and was supervised by the University's veterinarian. The study protocol was approved by the Veterinary Administration Medical Centre, Alexandroupolis, Greece.

\section{Bacteria preparation}

The Escherichia coli strain was the ATCC 35218 and was grown on McCongi agar (Becton Dickinson, Sparks, MD, USA) for $24 \mathrm{~h}$ at $35^{\circ} \mathrm{C}$. After it was grown, $1 \times 10^{10}$ Escherichia coli bacteria (in a $2-\mathrm{mL}$ volume of saline solution) were injected into the right pleural space.

\section{Empyema induction}

The rabbits were anaesthetised with ketamine; $50 \mathrm{mg} \cdot \mathrm{kg}^{-1}$ i.m. (Ketaset; Fort Dodge Laboratories Inc., Fort Dodge, IA, USA); atropine, $0.04 \mathrm{mg} \cdot \mathrm{kg}^{-1}$ (Demo SA, Athens, Greece); and xylazine, $5 \mathrm{mg} \cdot \mathrm{kg}^{-1}$ i.m. (Rompun; Bayer AG, Leverkusen). The right chest wall of each rabbit was shaved and then scrubbed with povidone-iodine (Betadine) and alcohol. The animals were placed in a supine position on an operating table, under a heating lamp, and a $0.5-\mathrm{cm}$ medial-to-lateral skin incision was made over the right anterior chest using a scalpel. A specially prepared 16-gauge angiocatheter (containing additional holes near the tip of the catheter) was then introduced into the pleural space. After placement of the catheter, any air within the pleural space was aspirated and the catheter was secured s.c. in the area between the scapulas [15]. The chest tubes were attached to a Heimlich valve with a three-way stopcock, in-line between the chest tube and the Heimlich valve.
Turpentine (1 mL) [16] (Riedel de Haen; Sigma-Aldrich Laborchemikalien, GMBH, Seelze Germany) was administered into the pleural space of the animals and the chest tube was then flushed with $1.5 \mathrm{~mL}$ of saline solution. Escherichia coli $\left(1 \times 10^{10}\right.$ in a final volume of $2 \mathrm{~mL}$ saline) was injected $24 \mathrm{~h}$ later through the cannula into the pleural cavity of the animals.

\section{Empyema verification}

A maximum of $0.5 \mathrm{~mL}$ of pleural fluid was removed for analysis at 12, 24, 48 and $72 \mathrm{~h}$ after bacterial injection. The $\mathrm{pH}$ of the pleural fluid was analysed using a blood gas machine [17] (Gem premier 3000, model 5700; Instrumentation laboratory, Lexington, MA, USA), and the glucose and lactate-D-hydrogenase (LDH) levels were analysed using a common microbiological laboratory (the upper limit of $\mathrm{LDH}$ in the present study's laboratory was $\left.460 \mathrm{U} \cdot \mathrm{L}^{-1}\right)$. An empyema was said to be present if the pleural fluid appeared grossly infected, if the glucose was $<40 \mathrm{mg} \cdot \mathrm{dL}^{-1}$ and if the pleural fluid $\mathrm{pH}$ was $<7.10$ and the $\mathrm{LDH}$ was $>1,000 \mathrm{U} \cdot \mathrm{L}^{-1}$ [15].

\section{Antibiotic administration}

After the presence of an empyema was verified, three rabbits were injected with moxifloxacin and three with levofloxacin, through their marginal ear vein over a 3-min period. The antibiotics injected $\left(25 \mathrm{mg} \cdot \mathrm{kg}^{-1}\right)$ were moxifloxacin (Octegra; Elpen Pharmaceutical Inc. Co., Greece) or levofloxacin (Tavanic; Aventis Pharma). Four animals served as controls and were infected with Escherichia coli but were not treated.

\section{Pleural fluid and blood specimens}

Blood and empyemic pleural fluid specimens were serially collected at 1, 4, 6 and $12 \mathrm{~h}$ after antibiotic administration, for the measurement of antibiotic levels at a later opportunity. Immediately after the specimens were collected, the blood and the pleural fluid samples were centrifuged at $850 \mathrm{~g}$ for $15 \mathrm{~min}$. Supernatants were then refrigerated at $-20^{\circ} \mathrm{C}$ overnight. The specimens were transported to the Dept of Morphology, Centre of Toxicology and Forensic Sciences Research, Medical School, University of Crete, Crete, Greece, the following morning using a refrigeration cooler with dry ice. Three rabbits were used for each antibiotic. For all antibiotics, duplicate specimens of blood and pleural fluid were obtained at each time point. The means of the duplicate values at each time point were used for the analysis.

\section{Measurement of antibiotic levels}

Chemicals. Methanol and acetonitrile (Hiper Solv BDH Laboratory Supplies, Poole, UK), water, orthophosphoric acid $85 \%$, potassium dihydrogen phosphate (E. Merck, Darmstadt, Germany) and triethylamine (Fluka Chemie, AG GH-9471; Buchs, Neu-Ulm, Germany) were used for the HPLC analysis.

Preparation of standard curves. Two separate stock solutions containing levofloxacin and moxifloxacin were prepared in water at $100 \mu \mathrm{g} \cdot \mathrm{mL}^{-1}$ and stored at $0^{\circ} \mathrm{C}$. From each of the initial stock solutions, nine diluted solutions containing levofloxacin and moxifloxacin were prepared at $0,0.065$, 
$0.125,0.25,0.5,1,2.5,5$ and $10 \mu \mathrm{g} \cdot \mathrm{mL}^{-1}$. The curve was linear for both drugs. Detection limits were defined by the concentration and were calculated for levofloxacin and moxifloxacin in the standard solutions at 0.065 and $0.125 \mu \mathrm{g} \cdot \mathrm{mL}^{-1}$, respectively.

Quantification of biological extracts. Blood samples from healthy animals were used as blind samples. An eight-point standard curve was prepared from the standard solutions for both drugs. The concentrations of the final fortified standards were $0,0.25,0.5,1,2.5,5,10$ and $25 \mu \mathrm{g} \cdot \mathrm{mL}^{-1}$. The curve was linear for both drugs and the limit of quantification, which gives accurate measurements, was calculated at $0.250 \mu \mathrm{g} \cdot \mathrm{mL}^{-1}$ for levofloxacin and moxifloxacin in the fortified solutions.

Apparatus. A reversed-phase HPLC method was carried out using a Kromasil 100, C18, 5- $\mu \mathrm{m}, 250 \mathrm{~mm} \times 4.6 \mathrm{~mm}$-ID column (Spectra Physics 8800; San Jose, CA, USA). The mobile phase for the determination of levofloxacin consisted of water: methanol:triethylamine (750:250:4) [18]. The $\mathrm{pH}$ of the mobile phase was adjusted to 3.2 with concentrated orthophosphoric acid. The mobile phase was filtered through a $0.20-\mu \mathrm{m}$ filter (Sarstedt Aktiengesellscafr \& Co., Numbrecht, Germany). The elution conditions were isocratic and the mobile phase flow rate was set at $1.0 \mathrm{~mL} \cdot \mathrm{min}^{-1}$. The mobile phase for the determination of moxifloxacin was a mixture of $10 \mathrm{mM}$ acetonitrile and potassium dihyrogenphosphate buffer (200:750 v:v) $[19,20]$. The $\mathrm{pH}$ was adjusted to 3.8 with concentrated orthophosphoric acid and the mobile phase was filtered through a $0.20-\mu \mathrm{m}$ filter. UV absorbance detection at $297 \mathrm{~nm}$ was carried out with a UV/Vis detector (Spectra Physics 8450) and the range of the detector was set at 0.01 AUFS. Under these conditions, the retention time of levofloxacin was $14.7 \mathrm{~min}$ and was $13.86 \mathrm{~min}$ for moxifloxacin.

Extraction procedure for HPLC analysis. The biological sample $(100 \mu \mathrm{L}$, blood or PF) was diluted with $200 \mu \mathrm{L}$ of methanol. The solution was vortex-mixed for $20 \mathrm{~s}$ and centrifuged for $5 \mathrm{~min}$ at 3,500 $\mathrm{g}$ to separate the precipitated proteins $[18,21]$. Supernatant $(50 \mu \mathrm{L})$ was injected into the column. The recovery of moxifloxacin and levofloxacin was calculated at $100.7 \%$ and $96.4 \%$, respectively.

\section{Necropsy of the rabbits}

The rabbits were sacrificed via a lethal dose of pentobarbital i.v. through the marginal ear vein at $96 \mathrm{~h}$. At autopsy, the thorax was bisected coronally, and the right and left hemithoraces were examined. Biopsy specimens from the parietal pleura with chest wall attached and visceral pleura with lung attached were placed in formalin for microscopic analysis.

\section{Statistical analysis}

Values are expressed as mean \pm SD unless otherwise indicated. The maximum of blood and pleural fluid concentrations (Cmax) and the time of peak concentration in blood and pleural fluid $(T \max )$ were taken directly from the respective curves. The areas under the time-concentration curves over $12 \mathrm{~h}$ (AUC) were estimated by trapezoidal rule. AUC:minimal inhibitory concentration (MIC) ratios, defined as the area under the inhibitory concentration curve and were calculated by dividing the AUC in the pleural fluid [22] by the MIC for the common strains in pleural empyema.

\section{Results}

All rabbits developed empyema after intrapleural injection of turpentine and $1 \times 10^{10}$ Escherichia coli (table 1). The pleural fluid was thick and grossly opaque. The pleural fluid $\mathrm{pH}$ was $<7.10$, the pleural fluid glucose level was $<40 \mathrm{mg} \cdot \mathrm{dL}^{-1}$ and the $\mathrm{LDH}$ was $>1,000 \mathrm{U} \cdot \mathrm{L}^{-1}$ in all rabbits (table 1 ). The pleural fluid culture results were positive in all rabbits (table 1).

The relationships between the pleural fluid antibiotic levels and the blood antibiotic levels varied from one antibiotic to the other (fig. 1). These relationships were primarily evaluated in two ways: first, the degree of penetration of the antibiotic

Table 1.-Pleural fluid findings in the rabbits immediately before administration of antibiotics

\begin{tabular}{lccccc}
\hline $\begin{array}{l}\text { Rabbit } \\
\text { no. }\end{array}$ & Antibiotic & $\mathrm{pH}$ & $\begin{array}{c}\mathrm{Glucose} \\
\mathrm{mg} \cdot \mathrm{dL}^{-1}\end{array}$ & $\mathrm{LDH} \mathrm{U} \cdot \mathrm{L}^{-1}$ & Culture \\
\hline 1 & Moxifloxacin & 6.93 & 17 & 2025 & Positive \\
2 & Moxifloxacin & 6.87 & 14 & 8307 & Positive \\
3 & Moxifloxacin & 6.97 & 12 & 3113 & Positive \\
1 & Levofloxacin & 7.06 & 15 & 1875 & Positive \\
2 & Levofloxacin & 7.01 & 26 & 1027 & Positive \\
3 & Levofloxacin & 7.09 & 8 & 2296 & Positive \\
1 & Control & 6.90 & 15 & 1100 & Positive \\
2 & Control & 7.1 & 32 & 1936 & Positive \\
3 & Control & 6.68 & 12 & 1965 & Positive \\
4 & Control & 7.02 & 34 & 2243 & Positive \\
\hline
\end{tabular}

LDH: lactate-D-hydrogenase.
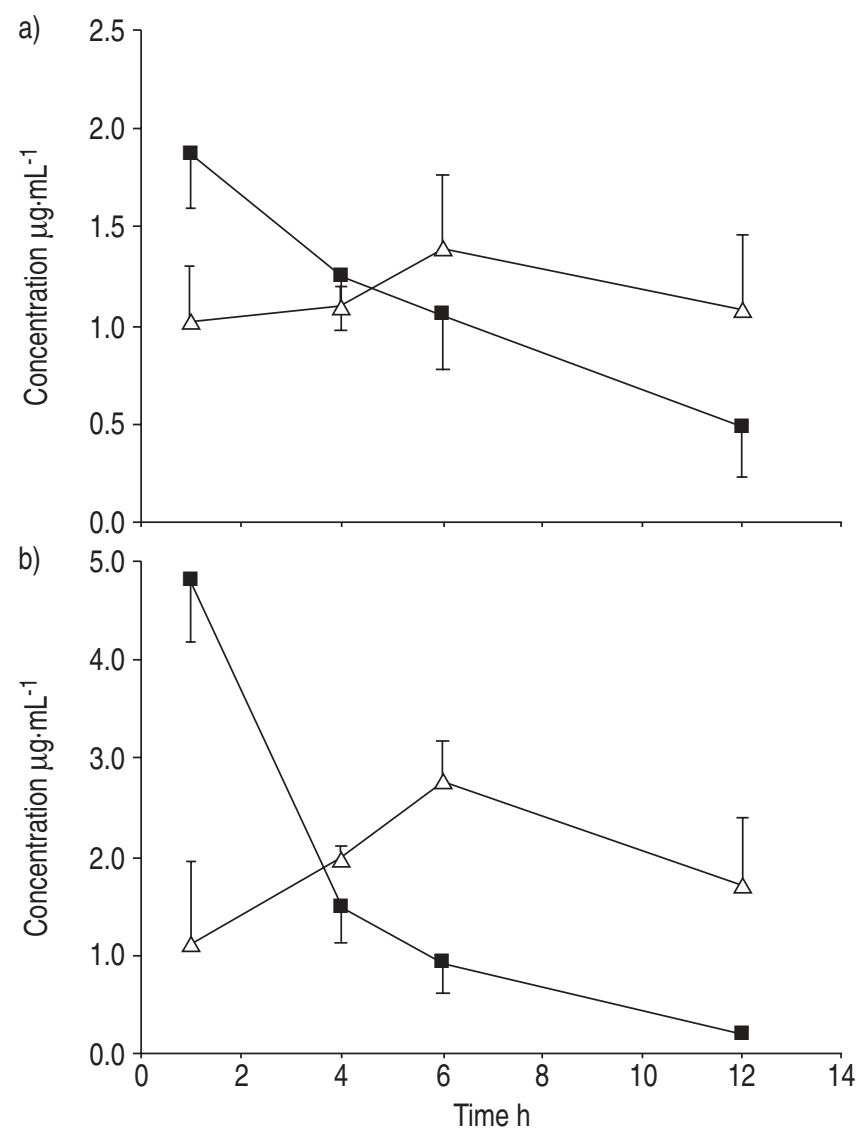

Fig. 1.-a) Levofloxacin and b) moxifloxacin concentration profiles in blood (ם) and pleural fluid $(\triangle)$ after i.v. administration. Data are presented as mean \pm SD for three rabbits. 
Table 2.-Summary of pharmacokinetic data for the two different antibiotics in the blood and the pleural fluid

\begin{tabular}{lcc}
\hline Variables & Moxifloxacin & Levofloxacin \\
\hline Dose $\mathrm{mg} \cdot \mathrm{kg}^{-1}$ & 25 & 25 \\
Peak blood level $\mu \mathrm{g} \cdot \mathrm{mL}^{-1}$ & 4.81 & 1.88 \\
Peak fluid level $\mu \mathrm{g} \cdot \mathrm{mL}^{-1}$ & 2.77 & 1.39 \\
PF/blood ratio & 0.5 & 0.7 \\
Tmax,blood h & 1 & 1 \\
Tmax,PF h & 6 & 6 \\
AUCPF/blood ratio & 1.37 & 1.13 \\
\hline
\end{tabular}

AUC: area under the curve for the first $12 \mathrm{~h}$; PF: pleural fluid; Tmax: time to maximum concentration.

into the pleural space and, secondly, the time of equilibration of blood and pleural fluid. The degree to which the different antibiotics penetrated into the pleural fluid was evaluated by comparing the AUCPF/AUCblood ratios of each antibiotic (table 2). Antibiotic penetration was greater for moxifloxacin (AUCPF/AUCblood ratio=1.37) than for levofloxacin (ratio= 1.13). The AUCPF/AUCblood ratios for moxifloxacin and levofloxacin were $>1$ because the pleural fluid levels remained elevated until $12 \mathrm{~h}$ despite decreasing blood levels.

The time to equilibration of the pleural fluid antibiotic level and the blood antibiotic level was more rapid for moxifloxacin $(3.9 \mathrm{~h})$ than for levofloxacin $(4.4 \mathrm{~h})$. With moxifloxacin, the peak pleural fluid antibiotic level occurred $6 \mathrm{~h}$ after infusion and decreased slowly thereafter. The pleural fluid level exceeded the blood level after the 3.9-h time point and was several times higher than the blood level at 6 and $12 \mathrm{~h}$.

With levofloxacin, the peak pleural fluid antibiotic level occurred $6 \mathrm{~h}$ after infusion. After the 6-h time point, it decreased slightly until the 12 -h time point. The pleural fluid level exceeded the blood level $4.4 \mathrm{~h}$ after infusion and was higher than the blood level at 6 and $12 \mathrm{~h}$.

The pharmacokinetic data for the two different antibiotics are compared in table 2. The higher ratio of the peak pleural fluid to the peak blood level occurred with levofloxacin. The time for the peak antibiotic level to occur in the pleural fluid was the same for levofloxacin and moxifloxacin $(6 \mathrm{~h})$.

\section{Pathological studies}

When the animals were sacrificed, the visceral and parietal pleural surfaces were thickened and multiple adhesions were present, which resulted in the formation of many pus pockets. Histological sections stained with haematoxylin-eosin showed prominent acute inflammatory cell infiltration.

\section{Discussion}

The present study, using an experimental model of empyema in the rabbit, shows that the penetration of moxifloxacin and levofloxacin into infected pleural fluid varies from one antibiotic to another.

Besides the AUCPF/blood ratio, the area under the inhibitory curve allows agents with long half-lives, like fluoroquinolones, to be compared with each other [23, 24]. For the most frequent microbes in pleural empyema, such as $H$. influenza, E. coli, Klebsiella spp., S. aureus and S. pneumoniae, a MIC of $0.03-0.25 \mu \mathrm{g} \cdot \mathrm{mL}^{-1}$ (moxifloxacin) or a MIC of $0.03-1 \mu \mathrm{g} \cdot \mathrm{mL}^{-1}$ (levofloxacin) is required [6], and the ratio AUC/MIC permits the studied quinolones to be contrasted.

STRAHILEVITZ et al. [22], studied the effect of moxifloxacin in an experimental pneumococcal pleural empyema model in rabbits. They reported that the AUC for moxifloxacin in the empyema fluid over $12 \mathrm{~h}$ was $34.3 \mathrm{mg} \cdot \mathrm{h}^{-1} \cdot \mathrm{L}^{-1}$, while the AUC in the present study was $22.9 \mu \mathrm{g} \cdot \mathrm{h}^{-1} \cdot \mathrm{mL}^{-1}$. In the study by STRAHILEVITZ et al. [22], the AUC in the blood was $29.4 \mathrm{mg} \cdot \mathrm{h}^{-1} \cdot \mathrm{L}^{-1}$, while in the current study it was $16.7 \mu \mathrm{g} \cdot \mathrm{h}^{-1} \cdot \mathrm{mL}^{-1}$. The reason that the AUC in the blood was higher in the study by STRAHILEVITZ et al. [22] is unclear, but this higher value for the AUC in the blood is the probable explanation for the lower AUC in the empyema fluid in the present study.

The AUCPF/blood ratio was 1.37 for moxifloxacin and 1.13 for levofloxacin, and the time to equilibration of the pleural fluid antibiotic level and the blood antibiotic level occurred faster with moxifloxacin (3.9 h) than with levofloxacin $(4.4 \mathrm{~h})$. In a previous study [14], the AUCPF/serum ratios were 0.98 for metronidazole, 2.31 for penicillin, 0.74 for clindamycin, 0.82 for ceftriaxone, 0.61 for vancomycin and 0.49 for gentamycin. In the same study [14], the time to equilibration was $2 \mathrm{~h}$ for metronidazole, $1 \mathrm{~h}$ for penicillin, $4.5 \mathrm{~h}$ for clindamycin, $4 \mathrm{~h}$ for ceftriaxone, $4 \mathrm{~h}$ for vancomycin and $3.5 \mathrm{~h}$ for gentamycin. The comparison to the above values demonstrates that the newer quinolones penetrate empyemic pleural fluid in a comparable manner to the older antibiotics.

Significant levels of moxifloxacin and levofloxacin remained in the pleural space for up to $12 \mathrm{~h}$, despite diminishing blood antibiotic levels over time. In previous studies, STRAHILEVITZ et al. [22] reported similar findings with moxifloxacin, while TEIXEIRA et al. [14] only measured levels up to $6 \mathrm{~h}$. However, they reported that levels of ceftriaxone were undetectable in the pleural fluid after $4 \mathrm{~h}$ [14].

Some previous studies have suggested that there is very little difference amongst antibiotics concerning their level of penetration into the pleural fluid. TARYLE et al. [25] studied 16 patients (including five with empyema), and concluded that antibiotic concentrations were usually $\geqslant 3 / 4$ of the concentrations in simultaneously obtained samples of serum (antibiotics reported in the study by TARYLE et al. [25] included penicillin, cephalothin, oxacillin, ampicillin, clindamycin and aminoglycosides). However, subsequent studies have shown that the penetration of aminoglycosides, particularly gentamicin, into the pleural fluid is poor if an empyema is present [26].

SAKUMA et al. [27] studied the penetration of intravenous antibiotics from the lung into the pleural space after pulmonary resection and concluded that higher doses of antibiotics are required in patients with empyema after pneumonectomy.

The findings of sustained elevated pleural fluid levels of moxifloxacin and levofloxacin in the current study were similar with that found for penicillin by TEIXEIRA et al. [14]. The pleural fluid antibiotic level remained $>1 \mu \mathrm{g} \cdot \mathrm{mL}^{-1}$ at $12 \mathrm{~h}$ after infusion, while the simultaneously obtained blood levels had diminished to lower levels. The reason for this is unclear. Perhaps the answer relates to the relationship between fluoroquinolones, and the increased permeability of the pleural space with inflammation or the increased acidity of pleural fluid with empyema. It may be advantageous to use quinolones for susceptible organisms in patients with empyema, due to their prolonged elevated levels in infected pleural fluid in this experimental model of empyema.

The equilibration between an antibiotic in the serum and the pleural fluid depends on several factors. These include the size of the pleural effusion (equilibration will occur less rapidly with larger pleural effusions), the thickness of the pleura (equilibration will occur less rapidly with a thicker pleura), the degree of pleural inflammation (equilibration will occur more rapidly with inflammation due to increased protein flux) and the antibiotic itself. In the present study, all of the above factors were maintained constant, with the only variable being the antibiotic.

There are certain limitations to the present study. It is not 
clear if these results obtained in the rabbit can be extrapolated to human, as rabbits are a species with thin visceral pleura, while humans have thick visceral pleura [28]. It is likely that the equilibration of antibiotics would be more rapid in species with thin pleura. Infusion of an antibiotic over a 3-min period is not a typical method for administration of these antibiotics in humans. This infusion method may have also led to changes in the equilibration curves, which would be different if a longer infusion time had been used. In the current study, antibiotic treatment was initiated relatively early in the course of empyema, $24 \mathrm{~h}$ after bacterial challenge. The equilibration might have been longer if the empyema had been present for several days. The repeated pleural fluid aspirations carried out in the present investigation could possibly be regarded as a partial pleural empyema drainage, facilitating recovery. However, it resembles more closely the current clinical approach to pleural empyema.

The present authors conclude that, in this rabbit model of empyema, there are differences in the degree of penetration into infected pleural fluid and in the equilibration time between serum and pleural fluid for the two quinolones. The clinical significance of these differences in humans is unknown, since there is not sufficient data concerning the penetration of these antimicrobial agents in the infected pleura in humans. More studies are needed to evaluate the pharmacokinetic parameters in the pleural space.

\section{References}

1. Light RW, Girard WM, Jenkinson SG. The incidence and significance of parapneumonic effusions. Am J Med 1980; 69: $507-512$

2. Bouros D, Hamm H. Infectious pleural effusions. In: Loddenkemper R, Antony VB, eds. Pleural Diseases. Eur Respir Monograph 2002; 22: 204-218.

3. Sasse S, Nguyen T, Teixeira LR, Light RW. The utility of daily therapeutic thoracentecis for the treatment of early empyema. Chest 1999; 116: 1703-1708.

4. Sasse S, Nguyen T, Mulligan M, Nai-San W, Mahutte K, Light RW. The effects of early chest tube placement on empyema resolution. Chest 1997; 111: 1679-1683.

5. Bouros D, Schiza S, Siafakas N. Fibrinolytics in the treatment of parapneumonic effusions. Monaldi Arch Chest Dis 1999; 54: 258-263.

6. Zhanel GG, Ennis K, Vercaigne L, et al. A critical review of the fluoroquinolones: focus on respiratory infections. Drugs 2002; 62: 13-59.

7. Detache CJ, Dewan N, O'Donahue WJ, et al. Clinical and economic considerations in the treatment of acute exacerbations of chronic bronchitis. J Antimicrob Chemother 1999; 43: Suppl., 107-113.

8. Siegel RE. Strategies for early discharge of the hospitalized patient with community-acquired pneumonia. Clin Chest Med 1999; 20: 599-605.

9. Palmer CS, Zhan C, Elixhauser A, et al. Economic assessment of the community-acquired pneumonia intervention trial employing levofloxacin. Clin Ther 2000; 22: 250264.

10. Davies BI, Maesen FP. Clinical effectiveness of levofloxacin in patients with acute purulent exacerbations of chronic bronchitis: the relationship with in-vitro activity. J Antimicrob Chemother 1999; 43: Suppl., 83-90.

11. Byl B, Jacobs F, Roucloux I, De Franquen P, Cappello M, Thys J-P. Penetration of meropenem in lung, bronchial mucosa and pleural tissues. Antimicrob Agents Chemother 1999; 43: 681-682.

12. Hamm H, Light RW. Parapneumonic effusion and empyema. Eur Respir J 1997; 10: 1150-1156.

13. Liapakis IE, Kottakis I, Light RW, Bouros D. Pharmacokinetics and pharmacodynamics in the pleural space. In: Bouros D, ed. Pleural Diseases. New York, Dekker 2004; pp 999-1008.

14. Teixeira LR, Sasse SA, Villarino MA, Nguyen T, Mulligan ME, Light RW. Antibiotic levels in empyemic pleural fluid. Chest 2000; 117: 1734-1739.

15. Sasse S, Causing L, Mulligan M. Serial pleural fluid analysis in a new experimental model of empyema. Chest 1996; 109: 1043-1048.

16. Sahn SA, Potts DE. Turpentine pleurisy in rabbits: a model of pleural fluid acidosis and low pleural fluid glucose. $\mathrm{Am}$ Rev Respir Dis 1978; 118: 893-901.

17. Chandler TM, McCoskey EH, Byrd RP Jr, Roy TM. Comparison of the use and accuracy of methods for determining pleural fluid $\mathrm{pH}$. South Med J 1999; 92: 214-217.

18. Bottcher S, Baum HV, Hoppe-Tichy T, Benz C, Sonntag HG. An HPLC assay and a microbiological assay to determine levofloxacin in soft tissue, bone, bile and serum. J Pharm Biomed Analysis 2001; 25: 197-203.

19. Lemoine $\mathrm{T}$, Breilh D, Ducint $\mathrm{D}$, et al. Determination of moxifloxacin (BAY 12-8039) in plasma and lung tissue by high-performance liquid chromatography with ultraviolet detection using a fully automated extraction method with a new polymeric cartridge. $J$ Chromatography $B$ 2000; 742: 247-254

20. Tsatsakis AM, Alegakis TK, Chalkiadakis GE, Askoxilakis I, Vlachonikolis I. Comparative pharmacokinetics of ceftriaxone after periincisional and intravenous administration in patients undergoing abdominal surgery. Eur J Pharm $S$ 1997; 5: 243-251.

21. Neckel U, Joukhadar C, Frossard M, Jager M, Muller M, Mayer BX. Simultaneous determination of levofloxacin and ciprofloxacin in microdialysates and plasma by highperformance liquid chromatography. Analytica Chimica Acta 2002; 463: 199-206.

22. Strahilevitz J, Lev A, Levi I, Fridman E, Rubinstein E. Experimental pneumococcal pleural empyema model: the effect of moxifloxacin. J Antimicrob Chemother 2003; 51: 665-669.

23. Schentag JJ, Gilliland KK, Paladino JA. What have we learned from the pharmacokinetic and pharmacodynamic theories? Clin Inf Dis 2001; 32: Suppl. 1, S39-S46.

24. Mouton JW, Dudley MN, Cars O, Derendorf H, Drusano GL. Standardization of pharmacokinetic/pharmacodynamic (PK/PD) terminology for anti-infective drugs. Int J Antimicrob Agents 2002; 19: 355-358.

25. Zhanel GG, Ennis K, Vercaigne L, et al. A critical review of the fluoroquinolones. Focus on respiratory tract infections. Drugs 2002; 62: 13-59.

26. Taryle DA, Good JT Jr, Morgan EJ III, et al. Antibiotic concentrations in human parapneumonic effusions. J Antimicrob Chemother 1981; 7: 171-177.

27. Thys JP, Vanderhoeft P, Herchuelz A, et al. Penetration of aminoglycosides in uninfected pleural exudates and in pleural empyemas. Chest 1988; 93: 530-532.

28. Sakuma T, Kubo H, Tanita T, Koike K, Fujimura S. Penetration of intravenous antibiotics from the lung into the pleural space after pulmonary resection. $J$ Thorac Cardiovasc Surg 1994; 107: 955-957.

29. Light RW. Pleural diseases, 4th Edn. Baltimore, MD, Williams and Wilkins, 2001; pp. 1-6. 